\title{
Rough Approximations for Incomplete Information*
}

\author{
Jun-Fang LUO \\ College of Mathematics \\ Southwest Jiaotong University \\ Chengdu, China
}

\author{
Ke-Yun QIN \\ College of Mathematics \\ Southwest Jiaotong University \\ Chengdu, China
}

\begin{abstract}
Rough set under incomplete information has been extensively studied. Based on valued tolerance relation for incomplete information system, several approaches were presented to dealing with the attribute reductions and rule extraction. We point out some drawbacks in the existing papers for valued tolerance relation based rough approximations and propose a new kind of rough approximation operators which is a generalization of Pawlak approximation operators for complete information system. Some basic properties of the approximation operators are investigated.
\end{abstract}

Keywords-Rough set; tolerance relation; valued tolerance relation

\section{INTRODUCTION}

The rough set theory (RST), proposed by Pawlak[7], is an effective tool for data analysis. It can be used in information system to describe the dependencies among attributes and evaluate the significance of attributes and derive decision rules. In an information system, each object in the universe is associated with some information that is characterized by a set of attributes. Objects characterized by the same information are indiscernible with the available information about them. Based on the indiscernibility relation, classical rough set theory has been used successfully in attribute reduction of information and decision systems.

In many practical situations, it may happen that the precise values of some of the attributes in an information system are not known, i.e. are missing or known partially. Such a system is called an incomplete information system. In order to deal with incomplete information systems, classical rough sets have been extended to several general models by using other binary relations or covers on the universe[1,8-10,14,16]. Based on these extended rough set models, the researchers have put forward several meaningful indiscernibility relations in incomplete information system to characterize the similarity of objects. For instance, Slowinski[11] proposed two different approaches to replace unknown value of attribute by specific subsets of values. Grzymala et al[2,3] performed computational studies on the medical data, where unknown values of attributes were replaced using probabilistic techniques. Kryszkiewicz introduced a kind of indiscernibility relation, called tolerance relation, to handle incomplete information tables[5,6]. Stefanowski[12] introduced two generalizations of the rough sets theory to handle the missing value. The first generalization introduces the use of a non symmetric similarity relation in order to formalize the idea of absent value semantics. The second proposal is based on the use of valued tolerance relations. A logical analysis and the computational experiments show that for the valued tolerance approach it is possible to obtain more informative approximations and decision rules than using the approach based on the simple tolerance relation. The tolerance relation has also been generalized to constrained similarity relation and constrained dissymmetrical similarity relation[4,13,15]. This paper is devoted to the discussion of valued tolerance relation based rough approximation operators. We pointed out that the lower (upper) approximability presented in [12] is not the generalization of Pawlak approximations. A new kind of lower (upper) approximability is proposed. Some basic properties are analyzed.

\section{SIMILARITY RELATION FOR INCOMPLETE INFORMATION TABLE}

Rough sets have been introduced by Pawlak[7] as an approach for analyzing vague information. Following Pawlak, an information table is a pair $I T=(U, A)$, where $U$ is a set of objects, $A$ is a set of attributes such that $\forall a \in A, a: U \rightarrow V_{a}, V_{a}$ is a domain of $a$ and $V=\cup_{a \in A} V_{a}$. Each subset of attributes $B \subseteq A$ defines an indiscernibility relation $\operatorname{IND}(B)$ as:

$$
\operatorname{IND}(B)=\{(x, y) \in U \times U ; \forall a \in B(a(x)=a(y))\}
$$

Clearly, $\operatorname{IND}(B)$ is an equivalence relation. Let $U / B$ be the family of all the equivalence classes of the equivalence relation $I N D(B)$. For each $X \subseteq U$, the lower and upper approximation of $X$ are defined by [7]:

$$
\begin{gathered}
\underline{B}(X)=\left\{x \in U ;[x]_{R} \subseteq X\right\}, \\
\bar{B}(X)=\left\{x \in U ;[x]_{R} \cap X \neq \varnothing\right\} .
\end{gathered}
$$

The rough set is characterized by its lower and upper approximations.

Let $I T=(U, A)$ be an incomplete information table. Kryszkiewicz[5] introduced the notion of tolerance relation. The key point in this approach is to interpret an unknown value of the attribute as similar to all other possible values for this attribute. Such an interpretation corresponds to the idea that such values are just missing, but they do exist. The tolerance relation $T_{B}$ with respect to $B \subseteq A$ is defined as [5]:

$$
T_{B}=\{(x, y) \in U \times U ; \forall a \in B(a(x)=a(y) \vee a(x)=* \vee a(y)=*)
$$

Clearly $T_{B}$ is a reflexive and symmetric relation, but not necessarily transitive. We denote by $T_{B}(x)$ the tolerance class

This work has been supported by the National Natural Science Foundation of China (Grant No. 61473239, 61175044) and The Fundamental Research Funds for the Central Universities of China (Grant No. 2682014ZT28). 
of $x$, that is $T_{B}(x)=\left\{y \in U ;(x, y) \in T_{B}\right\}$. The lower and upper approximations of $X \subseteq U$ are

$$
\begin{gathered}
\underline{T_{B}}(X)=\left\{x \in U ; T_{B}(x) \subseteq X\right\}, \\
\overline{T_{B}}(X)=\left\{x \in U ; T_{B}(x) \cap X \neq \varnothing\right\} .
\end{gathered}
$$

Stefanowski[12] introduced the absent values semantics for incomplete information tables. In this approach it is assumed that objects may be partially described not only because of our imperfect knowledge, but also because it is definitely impossible to describe them on all the attributes. The unknown values are not allowed to compare. Based on this point, the similarity relation $S_{B}$ is defined as:

$$
S_{B}=\{(x, y) \in U \times U ; \forall a \in B(a(x)=a(y) \vee a(x)=*)
$$

$S_{B}$ is a reflexive and transitive relation, but not necessarily symmetric. Based on $S_{B}$, the lower and upper approximations of $X \subseteq U$ are defined as:

$$
\begin{gathered}
\underline{S_{B}}(X)=\left\{x \in U ; S_{B}(x) \subseteq X\right\}, \\
\overline{S_{B}}(X)=\left\{x \in U ; S_{B}(x) \cap X \neq \varnothing\right\} .
\end{gathered}
$$

where $S_{B}(x)=\left\{y \in U ;(x, y) \in S_{B}\right\}$

In order to characterize incomplete information more precisely, Stefanowski[12] introduced the notion of valued tolerance relation. Let $a \in A$ be an attribute and $V_{a}$ the set of its known values. Given an object $x \in U$ with $a(x)=*$, the probability that $a(x)=e$ for any $e \in V_{a}$ is equal to $\left|V_{a}\right|^{-1}$. Moreover, if both values are unknown, then the probability that $x$ is similar to $y$ on the attribute is $\left|V_{a}\right|^{-2}$.Thus, the probability $R_{a}(x, y)$ for $x$ is similar to $y$ is defined by:

$$
R_{a}(x, y)=\left\{\begin{array}{c}
1 ; a(x)=a(y) \in V_{a} \\
0 ; a(x) \in V_{a} \wedge a(y) \in V_{a} \wedge a(x) \neq a(y) \\
\left|V_{a}\right|^{-1} ;\left(a(x)=* \wedge a(y) \in V_{a}\right) \vee\left(a(x) \in V_{a} \wedge a(y)=*\right) \\
\left|V_{a}\right|^{-2} ; a(x)=* \wedge a(y)=*
\end{array}\right.
$$

Let $R_{B}(x, y)=\prod_{a \in B} R_{a}(x, y)$. Based on $R_{B}(x, y)$, the $B$ - lower and the $B$ - upper approximability of $X$ by $\operatorname{set}^{Z}$ are defined as:

$$
\begin{aligned}
& \mu X_{B}(Z)=T_{z \in Z} T_{x \in \Theta_{B}(z)} I\left(R_{B}(z, x), x\right) \\
& \mu X^{B}(Z)=T_{z \in Z} S_{x \in \Theta_{B}(z)} T\left(R_{B}(z, x), x\right)
\end{aligned}
$$

where $\Theta_{B}(z)$ is the tolerance class of element $z, x$ is the membership degree of element $x$ in the set $X(x \in\{0,1\}), T, S$ and $I$ are t-norm, t-conorm and fuzzy implication respectively.

In this model, each subset of $U$ may be a lower or upper approximation of $X$, but to a different degree which is denote as lower (upper) approximability.
Theorem 1 Let $I T=(U, A)$ be an incomplete information table.

(1) If $Z_{1} \subseteq Z_{2}$, then $\mu X_{B}\left(Z_{1}\right) \geq \mu X_{B}\left(Z_{2}\right), \mu X^{B}\left(Z_{1}\right) \geq \mu X^{B}\left(Z_{2}\right)$.

(2) If $X_{1} \subseteq X_{2}$, then $\mu X_{1 B}(Z) \leq \mu X_{2 B}(Z), \mu X_{1}{ }^{B}(Z) \leq \mu X_{2}{ }^{B}(Z)$.

(3) If $B_{1} \subseteq B_{2}$, then $\mu X_{B_{1}}(Z) \leq \mu X_{B_{2}}$ (Z), $\mu X^{B_{1}}(Z) \geq \mu X^{B_{2}}(Z)$.

Proof: (1) By $T_{x \in \Theta_{B}(z)} I\left(R_{B}(z, x), x\right) \in[0,1]$ and $Z_{1} \subseteq Z_{2}$, we have

$$
\begin{aligned}
& \mu X_{B}\left(Z_{2}\right)=T_{z \in Z_{2}} T_{x \in \Theta_{B}(z)} I\left(R_{B}(z, x), x\right) \\
& =T_{z \in Z_{1}} T_{x \in \Theta_{B}(z)} I\left(R_{B}(z, x), x\right) \cdot T_{z \in Z_{2}-Z_{1}} T_{x \in \Theta_{B}(z)} I\left(R_{B}(z, x), x\right) \\
& \leq T_{z \in Z_{1}} T_{x \in \Theta_{B}(z)} I\left(R_{B}(z, x), x\right)=\mu X_{B}\left(Z_{1}\right)
\end{aligned}
$$

So we have $\mu X_{B}\left(Z_{1}\right) \geq \mu X_{B}\left(Z_{2}\right) . \mu X^{B}\left(Z_{1}\right) \geq \mu X^{B}\left(Z_{2}\right)$ can be proved similarly.

(2) Let $x_{1}$ be the membership degree of element in the set $X_{1}$ and $x_{2}$ be the membership degree of element in the set $X_{2}($ $\left.x_{1}, x_{2} \in\{0,1\} \quad\right)$. So $\mu X_{1 B}(Z)=T_{z \in Z} T_{x \in \Theta_{B}(z)} I\left(R_{B}(z, x), x_{1}\right) \quad$ and $\mu X_{2 B}(Z)=T_{z \in Z} T_{x \in \Theta_{B}(z)} I\left(R_{B}(z, x), x_{2}\right)$. By $X_{1} \subseteq X_{2}$, it follows that $I\left(R_{B}(z, x), x_{1}\right) \leq I\left(R_{B}(z, x), x_{2}\right)$. Consequently we have $\mu X_{1 B}(Z)$ $\leq \mu X_{2 B}(Z) \cdot \mu X_{1}^{B}(Z) \leq \mu X_{2}{ }^{B}(Z)$ can be proved similarly.

(3) By $B_{1} \subseteq B_{2}$ we have $\Theta_{B_{1}}(z) \supseteq \Theta_{B 2}(z)$ and $R_{B_{1}}(z, x) \geq$ $R_{B_{2}}(z, x)$. Thus

$$
\begin{aligned}
& \mu X_{B_{1}}(Z)=T_{z \in Z} T_{x \in \Theta_{B_{1}}(z)} I\left(R_{B_{1}}(z, x), x\right) \\
& =T_{z \in Z} T\left(T_{x \in \Theta_{B_{2}}(z)} I\left(R_{B_{1}}(z, x), x\right), T_{x \in \Theta_{B_{1}}(z)-\Theta_{B_{2}}(z)} I\left(R_{B_{1}}(z, x), x\right)\right) \\
& \leq T_{z \in Z} T_{x \in \Theta_{B_{2}}(z)} I\left(R_{B_{1}}(z, x), x\right) \\
& \leq T_{z \in Z} T_{x \in \Theta_{B_{2}}(z)} I\left(R_{B_{2}}(z, x), x\right)=\mu X_{B_{2}}(Z) . \\
& \mu X^{B_{2}}(Z)=T_{z \in Z} S_{x \in \Theta_{B_{2}}(z)} T\left(R_{B_{2}}(z, x), x\right) \\
& \leq T_{z \in Z} S_{x \in \Theta_{B_{1}}(z)} T\left(R_{B_{2}}(z, x), x\right) \\
& \leq T_{z \in Z} S_{x \in \Theta_{B_{1}}(z)} T\left(R_{B_{1}}(z, x), x\right)=\mu X^{B_{2}}(Z) .
\end{aligned}
$$

\section{ROUGH APPROXIMATIONS BASED ON VALUED TOLERANCE RELATION}

Let $I T=(U, A)$ be an incomplete information table. Intuitively, $R_{B}(x, y)$ is the similarity degree of $x$ and $y$ with respect to attribute set $B$. Clearly, $R_{B}(x, y) \in[0,1]$. If $D T=(U, A)$ is complete, then $R_{B}(x, y)$ will degenerate to indiscernibility relation $\operatorname{IND}(B)$. We note that the lower (upper) approximability will decrease with the increase of elements in $Z$. This does not coincide with the basic idea of Pawlak's rough set. In Pawlak rough set model, whether a set is lower 
(upper) approximation is definite. It does not happen that, the smaller the set, the more possible it is lower (upper) approximation. Actually, (5) and (6) are based on the observation that, in classical rough set,

$$
\begin{gathered}
Z=X_{B} \Rightarrow \forall z \in Z\left(\Theta_{B}(z) \subseteq X\right), \\
Z=X^{B} \Rightarrow \forall z \in Z\left(\Theta_{B}(z) \cap X \neq \varnothing\right),
\end{gathered}
$$

where $X_{B}$ and $X^{B}$ are lower and upper approximations of $X$ respectively. It is worth noticing that this is a necessary condition but not sufficient. Actually, we have

Theorem 2 Let $I T=(U, A)$ be an incomplete information table, $B \subseteq A$.

(1) $Z=X_{B}$ if and only if $\forall z \in Z\left(\Theta_{B}(z) \subseteq X\right) \wedge \forall z \in \sim Z$ $\left(\Theta_{B}(z) \cap \sim X \neq \varnothing\right)$.

(2) $Z=X^{B}$ if and only if $\forall z \in Z\left(\Theta_{B}(z) \cap X \neq \varnothing\right) \wedge \forall z \in \sim Z$ $\left(\Theta_{B}(z) \subseteq \sim X\right)$.

Proof: (1) Let $Z=X_{B}$. For each $z \in Z$, we have $\Theta_{B}(z) \subseteq X$ by $z \in X_{B}$. Furthermore, for each $z \in \sim Z$, we have $\Theta_{B}(z) \not \subset X$ by $z \notin X_{B}$. Thus $\Theta_{B}(z) \cap \sim X \neq \varnothing$. Conversely, for each $z \in Z$, by $\Theta_{B}(z) \subseteq X$ we have $z \in X_{B}$ and hence $Z \subseteq X_{B}$. Furthermore, for each $z \in X_{B}$ we have $\Theta_{B}(z) \subseteq X$ and hence $\Theta_{B}(z) \cap \sim X=\varnothing$. It follows that $z \notin \sim Z$ and thus $z \in Z$. Consequently, $X_{B} \subseteq Z$.

(2) can be proved similarly.

Based on this theorem, we propose the following definition.

Definition 1 Let $I T=(U, A)$ be an incomplete information table, $B \subseteq A$.The $B$ - lower approximability $\gamma X_{B}(Z)$ and the $B$ - upper approximability $\gamma X^{B}(Z)$ of $X$ by set $Z$ are defined as:

$$
\begin{aligned}
& \gamma X_{B}(Z)=T\left(\mu X_{B}(Z), \delta X_{B}(Z)\right), \\
& \gamma X^{B}(Z)=T\left(\mu X^{B}(Z), \delta X^{B}(Z)\right),
\end{aligned}
$$

Where

$$
\begin{gathered}
\delta X_{B}(Z)=T_{z \in U-Z} S_{x \in \Theta_{B}(z)} T\left(R_{B}(z, x), 1-x\right), \\
\delta X^{B}(Z)=T_{z \in U-Z} T_{x \in \Theta_{B}(z)} I\left(R_{B}(z, x), 1-x\right) .
\end{gathered}
$$

Theorem 3 Let $I T=(U, A)$ be an incomplete information table, and $B \subseteq A, X, Z \subseteq U$.

(1) $\gamma X_{B}(Z)=\gamma(\sim X)^{B}(\sim Z)$.

(2) $\gamma X^{B}(Z)=\gamma(\sim X)_{B}(\sim Z)$.

Theorem 4 Let $I T=(U, A)$ be a complete information table and $B \subseteq A, X, Z \subseteq U$.

(1) $Z=X_{B}$ if and only if $\gamma X_{B}(Z)=1$.
(2) $Z=X^{B}$ if and only if $\gamma X^{B}(Z)=1$.

Proof: (1) For complete information table, $R_{B}(z, x) \in\{0,1\}$. Let $Z=X_{B}$. For each $z \in Z$, we have $\Theta_{B}(z) \subseteq X$, where $\Theta_{B}(z)$ is the equivalence class containing $z$. For any $x \in \Theta_{B}(z)$, it follows that $x \in X$, and hence $x=1$. Thus $I\left(R_{B}(z, x), x\right)=1 \quad$ and hence $\quad \mu X_{B}(Z)=\quad T_{z \in Z} T_{x \in \Theta_{B}(z)}$ $I\left(R_{B}(z, x), x\right)=1$.

For each $z \in U-Z$, it follows that $z \notin X_{B}$ and thus $\Theta_{B}(z) \not \subset X$. There exists $x \in \Theta_{B}(z)$ such that $x \notin X$. Thus $R_{B}(z, x)=1-x=1$ and $\left.T\left(R_{B}(z, x), 1-x\right)\right)=1$. Consequently we have $\delta X_{B}(Z)=T_{z \in U-Z} S_{x \in \Theta_{B}(z)} T\left(R_{B}(z, x), 1-x\right)=1$. So, $\gamma X_{B}(Z)=1$ as required.

Conversely, assume that $\gamma X_{B}(Z)=1$. It follows that $\mu X_{B}(Z)=\delta X_{B}(Z)=1$. For each $z \in Z$ and $x \in \Theta_{B}(z)$, we have $1=I\left(R_{B}(z, x), x\right)=I(1, x)$ and hence $x=1$. That is $x \in X$ and $\Theta_{B}(z) \subseteq X$. Thus $Z \subseteq X_{B}$. On the other hand, for each $z \in U-Z$, by $S_{x \in \Theta_{B}(z)} T\left(R_{B}(z, x), 1-x\right)=1$ it follows that there exists $x \in \Theta_{B}(z)$ such that $T\left(R_{B}(z, x), 1-x\right)=1$. Thus $x=0$ and $x \notin X$. So we have $\Theta_{B}(z) \not \subset X$ and $z \notin X_{B}$. Consequently $X_{B} \subseteq Z$ and $Z=X_{B}$ as required.

(2) Let $Z=X^{B}$. For each $z \in Z$, we have $\Theta_{B}(z) \cap X \neq \varnothing$, where $\Theta_{B}(z)$ is the equivalence class containing $z$. Hence there exists $x \in \Theta_{B}(z)$ such that $x \in X$. Thus $T\left(R_{B}(z, x), 1\right)=1$ and $\quad S_{x \in \Theta_{B}(z)} T\left(R_{B}(z, x), x\right)=1 \quad$. So $\mu X^{B}(Z)=T_{z \in Z} S_{x \in \Theta_{B}(z)}$ $T\left(R_{B}(z, x), x\right)=1$. For each $z \in U-Z$, it follows that $z \notin X^{B}$ and thus $\Theta_{B}(z) \cap X=\varnothing$, i.e. $\Theta_{B}(z) \subseteq \sim X$. So, for any $x \in \Theta_{B}(z)$, we have $x \in \sim X$. Thus $I\left(R_{B}(z, x), 1-x\right)=1$ and hence $\delta X^{B}(Z)=$ $T_{z \in U-Z} T_{x \in \Theta_{B}(z)} I\left(R_{B}(z, x), 1-x\right)=1$.

So, $\gamma X^{B}(Z)=1$ as required.

Conversely, assume that $\gamma X^{B}(Z)=1$. It follows that $\mu X^{B}(Z)=T_{z \in Z} S_{x \in \Theta_{B}(z)} T\left(R_{B}(z, x), x\right)=1 \quad$ and $\quad \delta X^{B}(Z)=T_{z \in U-Z}$ $T_{x \in \Theta_{B}(z)} I\left(R_{B}(z, x), 1-x\right)=1$. For each $z \in Z$, there exists $x \in \Theta_{B}(z)$ such that $T\left(R_{B}(z, x), x\right)=1$. Thus $x=1$ and $x \in X$. So we have $\Theta_{B}(z) \cap X \neq \varnothing$. Thus $z \in X^{B}$ and $Z \subseteq X^{B}$. On the other hand, for each $z \notin Z$ and $x \in \Theta_{B}(z)$, we have $I\left(R_{B}(z, x), 1-x\right)=1$. It follows that $x \notin X$ and $\Theta_{B}(z) \cap X=\varnothing$. So $z \notin X^{B}$. Thus $X^{B} \subseteq Z$.Consequently $X^{B}=Z$.

This theorem shows that Definition 1 is a generalization of Pawlak approximation operators. In what follows, we take $T(x, y)=x y, S(x, y)=x+y-x y$ and $I(x, y)=1-x+x y$.

Theorem 5 Let $I T=(U, A)$ be an incomplete information table, and $B \subseteq A, X, Z \subseteq U$. 

(1) $\mu X_{B}(Z)=\prod_{z \in Z} \prod_{x \in \Theta_{B}(z) \cap(\sim X)}\left(1-R_{B}(z, x)\right)$.
(2) $\mu X^{B}(Z)=\prod_{z \in Z}\left(1-\prod_{x \in \Theta_{B}(z) \cap X}\left(1-R_{B}(z, x)\right)\right)$.
(3) $\delta X_{B}(Z)=\prod_{z \in U-Z}\left(1-\prod_{x \in \Theta_{B}(z) \cap(\sim X)}\left(1-R_{B}(z, x)\right)\right)$.
(4) $\delta X^{B}(Z)=\prod_{z \in U-Z} \prod_{x \in \Theta_{B}(z) \cap X}\left(1-R_{B}(z, x)\right)$.

Proof: (2) For each $z \in Z$, and $x \in \Theta_{B}(z), x \in X$ implies $T\left(R_{B}(z, x), x\right)=R_{B}(z, x)$ and $x \in U-X$ implies $T\left(R_{B}(z, x), x\right)=0$. Thus

$$
S_{x \in \Theta_{B}(z)} T\left(R_{B}(z, x), x\right)=S_{x \in \Theta_{B}(z) \cap X} R_{B}(z, x)=1-\prod_{x \in \Theta_{B}(z) \cap X}\left(1-R_{B}(z, x)\right) .
$$

Consequently, we have

$$
\mu X^{B}(Z)=\prod_{z \in Z}\left(1-\prod_{x \in \Theta_{B}(z) \cap X}\left(1-R_{B}(z, x)\right)\right) .
$$

(1), (3) and (4) can be proved similarly.

Corollary 1 Let $I T=(U, A)$ be an incomplete information table, and $B \subseteq A, X, Z \subseteq U$.

$$
\begin{aligned}
& \text { (1) } \mu X_{B}(Z)=\delta(\sim X)^{B}(\sim Z) . \\
& \text { (2) } \mu X^{B}(Z)=\delta(\sim X)_{B}(\sim Z) .
\end{aligned}
$$

Theorem 6 Let $I T=(U, A)$ be an incomplete information table.

(1)If $Z_{1} \subseteq Z_{2}$, then $\delta X_{B}\left(Z_{1}\right) \leq \delta X_{B}\left(Z_{2}\right), \delta X^{B}\left(Z_{1}\right) \leq \delta X^{B}\left(Z_{2}\right)$.

(2)If $X_{1} \subseteq X_{2}$, then $\delta X_{1 B}(Z) \geq \delta X_{2 B}(Z), \delta X_{1}^{B}(Z) \geq \delta X_{2}{ }^{B}(Z)$.

(3)If $B_{1} \subseteq B_{2}$, then $\delta X_{B_{1}}(Z) \geq \delta X_{B_{2}}(Z), \delta X^{B_{1}}(Z) \leq \delta X^{B_{2}}(Z)$.

Proof: (1) By Theorem 1 and Corollary 1,

$$
\begin{aligned}
& \delta X_{B}\left(Z_{1}\right)=\mu(\sim X)^{B}\left(\sim Z_{1}\right) \leq \mu(\sim X)^{B}\left(\sim Z_{2}\right)=\delta X_{B}\left(Z_{2}\right) \\
& \delta X^{B}\left(Z_{1}\right)=\mu(\sim X)_{B}\left(\sim Z_{1}\right) \leq \mu(\sim X)_{B}\left(\sim Z_{2}\right)=\delta X^{B}\left(Z_{2}\right)
\end{aligned}
$$

(2),(3)can be proved similarly.

Theorem 7 Let $I T=(U, A)$ be an incomplete information table, and $B \subseteq A, X \subseteq U$.

$$
\begin{aligned}
& \text { (1) } \gamma X_{B}(X)=\prod_{z \in X} \prod_{x \in \Theta_{B}(z) \cap(\sim X)}\left(1-R_{B}(z, x)\right) . \\
& \text { (2) } \gamma X^{B}(X)=\prod_{z \in \sim X} \prod_{x \in \Theta_{B}(z) \cap X}\left(1-R_{B}(z, x)\right) .
\end{aligned}
$$

Proof: (1) For each $z \in U-X$, we have $z \in \Theta_{B}(z) \cap(\sim X)$ and $1-R_{B}(z, z)=0$. Thus $\prod_{x \in \Theta_{B}(z) \cap(\sim X)}\left(1-R_{B}(z, x)\right)=0$ and hence $\delta X_{B}(X)=\prod_{z \in U-X}\left(1-\prod_{x \in \Theta_{B}(z) \cap(\sim X)}\left(1-R_{B}(z, x)\right)\right)=1$.

Consequently we have

$$
\gamma X_{B}(X)=\mu X_{B}(X)=\prod_{z \in X} \prod_{x \in \Theta_{B}(z) \cap(\sim X)}\left(1-R_{B}(z, x)\right)
$$

(2) can be proved similarly.

Theorem 8 Let $I T=(U, A)$ be an incomplete information table, and $B \subseteq A, X_{1}, X_{2}, Z_{1}, Z_{2} \subseteq U$.

(1) $\mu\left(X_{1} \cap X_{2}\right)_{B}\left(Z_{1} \cap Z_{2}\right) \geq \mu X_{1 B}\left(Z_{1}\right) \cdot \mu X_{2 B}\left(Z_{2}\right)$.

(2) $\delta\left(X_{1} \cap X_{2}\right)_{B}\left(Z_{1} \cap Z_{2}\right) \geq \delta X_{1 B}\left(Z_{1}\right) \cdot \delta X_{2 B}\left(Z_{2}\right)$.

(3) $\gamma\left(X_{1} \cap X_{2}\right)_{B}\left(Z_{1} \cap Z_{2}\right) \geq \gamma X_{1 B}\left(Z_{1}\right) \cdot \gamma X_{2 B}\left(Z_{2}\right)$.

Proof: (1) Let $z \in Z_{1} \cap Z_{2}$, by $\Theta_{B}(z) \cap\left(\sim X_{1} \cup \sim X_{2}\right)=$ $\left(\Theta_{B}(z) \cap \sim X_{1}\right) \cup\left(\Theta_{B}(z) \cap \sim X_{2}\right)$,

$$
\begin{aligned}
& \prod_{x \in \Theta_{B}(z) \sim \sim X_{1}}\left(1-R_{B}(z, x)\right) \cdot \prod_{x \in \Theta_{B}(z) \cap \sim X_{2}}\left(1-R_{B}(z, x)\right) \\
= & \prod_{x \in \Theta_{B}(z) \cap\left(\sim X_{1} \cup \sim X_{2}\right)}\left(1-R_{B}(z, x)\right) \cdot \prod_{x \in \Theta_{B}(z) \cap \sim X_{1} \cap \sim X_{2}}\left(1-R_{B}(z, x)\right) \\
\leq & \prod_{x \in \Theta_{B}(z) \cap\left(\sim X_{1} \cup \sim X_{2}\right)}\left(1-R_{B}(z, x)\right),
\end{aligned}
$$

It follows that

$$
\begin{aligned}
& \mu X_{1 B}\left(Z_{1}\right) \cdot \mu X_{2 B}\left(Z_{2}\right) \\
& =\prod_{z \in Z_{1}} \prod_{x \in \Theta_{B}(z) \cap\left(\sim X_{1}\right)}\left(1-R_{B}(z, x)\right) \cdot \prod_{z \in Z_{2}} \prod_{x \in \Theta_{B}(z) \cap\left(\sim X_{2}\right)}\left(1-R_{B}(z, x)\right) \\
& \leq \prod_{z \in Z_{1} \cap Z_{2} x \in \Theta_{B}(z) \cap\left(\sim X_{1}\right)}\left(1-R_{B}(z, x)\right) \cdot \prod_{z \in Z_{1} \cap Z_{2}} \prod_{x \in \Theta_{B}(z) \cap\left(\sim X_{2}\right)}\left(1-R_{B}(z, x)\right) \\
& =\prod_{z \in Z_{1} \cap Z_{2}}\left(\prod_{x \in \Theta_{B}(z) \cap\left(\sim X_{1}\right)}\left(1-R_{B}(z, x)\right) \cdot \prod_{x \in \Theta_{B}(z) \cap\left(\sim X_{2}\right)}\left(1-R_{B}(z, x)\right)\right) \\
& \leq \prod_{z \in Z_{1} \cap Z_{2}} \prod_{x \in \Theta_{B}(z) \cap\left(\sim X_{1} \cup \sim X_{2}\right)}\left(1-R_{B}(z, x)\right)=\mu\left(X_{1} \cap X_{2}\right)_{B}\left(Z_{1} \cap Z_{2}\right)
\end{aligned}
$$

(2) For each $z \in Z_{1} \cap Z_{2}$, by

$$
\begin{aligned}
& \delta X_{1 B}\left(Z_{1}\right) \cdot \delta X_{2 B}\left(Z_{2}\right) \\
& =\prod_{z \in \sim Z_{1}}\left(1-\prod_{x \in \Theta_{B}(z) \cap\left(\sim X_{1}\right)}\left(1-R_{B}(z, x)\right)\right) \cdot \prod_{z \in \sim Z_{2}}\left(1-\prod_{x \in \Theta_{B}(z) \cap\left(\sim X_{2}\right)}\left(1-R_{B}(z, x)\right)\right) \\
& \leq \prod_{z \in \sim Z_{1}}\left(1-\prod_{x \in \Theta_{B}(z) \cap\left(\sim X_{1} \cup \sim X_{2}\right)}\left(1-R_{B}(z, x)\right)\right) \cdot \prod_{z \in Z_{2}}\left(1-\prod_{x \in \Theta_{B}(z) \cap\left(\sim X_{1} \cup \sim X_{2}\right)}\left(1-R_{B}(z, x)\right)\right) \\
& =\prod_{z \in \sim Z_{1} \cup \sim Z_{2}}\left(1-\prod_{x \in \Theta_{B}(z) \cap\left(\sim X_{1} \cup \sim X_{2}\right)}\left(1-R_{B}(z, x)\right)\right) \cdot \prod_{z \in \sim Z_{1} \sim Z_{2}}\left(1-\prod_{\left.x \in \Theta_{B}(z) \sim \sim \sim X_{1} \cup \sim X_{2}\right)}\left(1-R_{B}(z, x)\right)\right) \\
& \leq \prod_{z \in Z_{1} \cup \sim Z_{2}}\left(1-\prod_{x \in \Theta_{B}(z) \wedge\left(\sim X_{1} \cup \sim X_{2}\right)}\left(1-R_{B}(z, x)\right)\right)=\delta\left(X_{1} \cap X_{2}\right)_{B}\left(Z_{1} \cap Z_{2}\right)
\end{aligned}
$$

(3) is straightforward from (1) and (2).

Corollary 2 Let $I T=(U, A)$ be an incomplete information table, and $B \subseteq A, X_{1}, X_{2}, Z_{1}, Z_{2} \subseteq U$.
(1) $\mu\left(X_{1} \cup X_{2}\right)^{B}\left(Z_{1} \cup Z_{2}\right) \geq \mu X_{1}^{B}\left(Z_{1}\right) \cdot \mu X_{2}^{B}\left(Z_{2}\right)$.
(2) $\delta\left(X_{1} \cup X_{2}\right)^{B}\left(Z_{1} \cup Z_{2}\right) \geq \delta X_{1}{ }^{B}\left(Z_{1}\right) \cdot \delta X_{2}{ }^{B}\left(Z_{2}\right)$.
(3) $\gamma\left(X_{1} \cup X_{2}\right)^{B}\left(Z_{1} \cup Z_{2}\right) \geq \gamma X_{1}^{B}\left(Z_{1}\right) \cdot \gamma X_{2}^{B}\left(Z_{2}\right)$.

Proof: (1) By Theorem 8 and Corollary 1,

$$
\mu\left(X_{1} \cup X_{2}\right)^{B}\left(Z_{1} \cup Z_{2}\right)=\delta\left(\sim X_{1} \cap \sim X_{2}\right)_{B}\left(\sim Z_{1} \cap \sim Z_{2}\right)
$$




$$
\geq \delta\left(\sim X_{1}\right)_{B}\left(\sim Z_{1}\right) \cdot \delta\left(\sim X_{2}\right)_{B}\left(\sim Z_{2}\right)=\mu X_{1}^{B}\left(Z_{1}\right) \cdot \mu X_{2}{ }^{B}\left(Z_{2}\right)
$$

(2) and (3) can be proved similarly.

\section{CONCLUSIONS}

Rough set under incomplete information has been extensively studied. For incomplete information system, researchers have put forward several similarity relations, such as tolerance relation, non-symmetric relation, valued tolerance relation etc. Based on valued tolerance relation, we proposed a new kind of rough approximation operators which is a generalization of Pawlak approximation operators for complete information system. Some basic properties of the approximation operators are investigated. Based on this work, we can further probe the rough set model under incomplete information and its application in knowledge discovery.

\section{REFERENCES}

[1] Z.Bonikowski, E.Bryniarski, U.Wybraniec, "Extensions and intentions in the rough set theory," Information Sciences, vol. 107, pp. 149-167, 1998.

[2] J.W.Grzymala-Busse, W.Grzymala-Busse, L.K.Goodwin, A closest fit approach to missing attribute values, Proc. 7th Workshop New Direction in Rough Sets, Data Mining, and Granular-Soft Computing Yamaguchi, Japan, Springer Verlag LNAI 1711, 1999, pp. 405-414.

[3] J.W.Grzymala-Busse, M.Hu, A comparison of several approaches to missing attribute values in data mining, Proceedings of the 2nd Int. Conference on Rough Sets and New Trends in Computing, Banff 2000, pp. 340-347.

[4] L.H.Guan, G.Y.Wang, Generalized approximations defined by nonequivalence relations, Information Sciences, vol. 193, pp. 163-179,
2012.

[5] M.Kryszkiewicz, Rough set approach to incomplete information system, Information Sciences, vol. 112, pp. 39-49, 1998.

[6] M.Kryszkiewicz, Properties of incomplete information systems in the framework of rough sets, Rough Sets in Data Mining and Knowledge Discovery, Physica-Verlag, 1998, pp. 422-450.

[7] Z.Pawlak, Rough sets, Int. J. Computer and Information Sci., vol. 11, pp. 341-356, 1982.

[8] Z.Pawlak, A.Skowron, Rough sets: Some extensions, Information Sciences, vol. 177, pp. 28-40, 2007.

[9] K.Qin, Z.Pei, J.Yang, Y.Xu, Approximation operators on complete completely distributive lattices, Information Sciences, vol. 247, pp. 123130,2013

[10] A.M.Radzikowska, E.E.Kerre, A comparative study of fuzzy rough sets, Fuzzy Sets and Systems, vol. 126, pp.137-155, 2002

[11] R.Slowinski, J.Stefanowski, Rough classification in incomplete information systems, Math. Computing Modeling, 12 (10/11), 1989, pp. 1347-1357.

[12] J.Stefanowski, A.Tsoukias, Incomplete information tables and rough classification, Computational Intelligence, 17(3)2001, pp. 545-566

[13] G.Y.Wang, Extension of rough set under incomplete information systems, Journal of Computer Research and Development (in Chinese), vol. 39, pp. 1238-1243, 2002.

[14] Y.Y.Yao, Relational interpretation of neighborhood operators and rough set approximation operator, Information Sciences, 111, pp.239-259, 1998.

[15] X.Yin, X.Jia, L.Shang, A new extension model of rough sets under incomplete information, Lecture Notes in Artificial Intelligence, 4062, pp. 141-146, 2006.

[16] X.H.Zhang, B.Zhou, P.Li, A general frame for intuitionistic fuzzy rough sets, Information Sciences, 216, pp.34-49, 2012. 